\title{
A importância do Fisioterapeuta no controle e na prevenção do Diabetes Mellitus Gestacional: Uma breve revisão integrativa
}

The importance of the Physiotherapist in the control and prevention of Gestational Diabetes

\author{
Mellitus: A brief integrative review
}

La importância del Fisioterapeuta em el control y prevención de la Diabetes Mellitus Gestacional:

Una breve revisión integradora

Recebido: 03/03/2021 | Revisado: 10/03/2021 | Aceito: 22/03/2021 | Publicado: 30/03/2021

Brinia Dantas de Araujo

ORCID: https://orcid.org/0000-0002-3077-1856 Faculdade de Comunicação e Turismo de Olinda, Brasil

E-mail: briniaadantas@gmail.com

Mirelle Vasconcelos Freitas

ORCID: https://orcid.org/0000-0002-3745-9443

Faculdade de Comunicação e Turismo de Olinda, Brasil

E-mail: mirelle.vf17@gmail.com

Dandara Cristine Rodrigues Falcão

ORCID: https://orcid.org/0000-0002-8464-7818

Faculdade de Comunicação e Turismo de Olinda, Brasil

E-mail: dandara.crf25@gmail.com

Letícia Viriato de Albuquerque

ORCID: https://orcid.org/0000-0002-4096-2752

Faculdade de Comunicação e Turismo de Olinda, Brasil

E-mail: leticiaviriato98@gmail.com

Jailson Dias de Vasconcelos

ORCID: https://orcid.org/0000-0002-5498-7803

Faculdade de Comunicação e Turismo de Olinda, Brasil

E-mail: jailsonbessan2011@ hotmail.com

Clarisse Mariana Juvencio de Siqueira

ORCID: https://orcid.org/0000-0001-5099-3360

Faculdade de Comunicação e Turismo de Olinda, Brasil

E-mail: clarissesqr@gmail.com

Marcella Maria da Silva

ORCID: https://orcid.org/0000-0003-3965-5736

Faculdade de Comunicação e Turismo de Olinda, Brasil

E-mail: adlaynneh@gmail.com

Stephane Roseno Trajano da Silva

ORCID: https://orcid.org/0000-0002-6174-0382

Universidade Federal de Pernambuco, Brasil

E-mail: stephane.trajano@ufpe.com

Tatiana Imbellone de Barros

ORCID: https://orcid.org/0000-0001-9250-6989

Faculdade de Comunicação e Turismo de Olinda, Brasil

E-mail: thatyannaimbelloni@gmail.com

Verônica Amorim Celestino

ORCID: https://orcid.org/0000-0002-0787-0290

Faculdade de Comunicação e Turismo de Olinda, Brasil

E-mail: veraacelestino_@hotmail.com

Gisele Nascimento de Santana

ORCID: https://orcid.org/0000-0003-2839-8615

Faculdade de Comunicação e Turismo de Olinda, Brasil

E-mail: giselenascimento30@hotmail.com

Evilâne Dias Barral

ORCID: https://orcid.org/0000-0002-3261-6817

Faculdade de Comunicação e Turismo de Olinda, Brasil

E-mail: elielza_dias578@outlook.com

Etiene da Silva Andrade

ORCID: https://orcid.org/0000-0001-9376-1631 Instituto Paiva, Brasi

E-mail: etiene.pos.fisio@gmail.com 


\title{
Resumo
}

Introdução: O Diabetes Mellitus Gestacional (DMG) ocorre quando o corpo não é eficiente para captar glicose suficiente para realizar as necessidades extras durante a gestação, resultando em hiperglicemia de gravidade variável, proveniente do déficit total ou parcial do hormônio insulina. Objetivo: Realizar uma revisão da literatura sobre a importância do fisioterapeuta no controle e na prevenção do Diabetes Mellitus Gestacional. Métodos: Trata-se de uma revisão da literatura. Foi realizado levantamento de dados nas bases PEDro, PubMed e Cochrane Library. Os descritores selecionados foram: Eclampsia, Fisioterapia, e Diabetes Gestacional. Os critérios de inclusão foram artigos completos, publicadas em periódicos nacionais e internacionais, e que contivessem informações que respondessem a temática do estudo. Os critérios de exclusão envolveram as publicações duplicadas. Resultados e Discussões: No cruzamento dos descritores, foram encontrados 17 artigos, destes, 4 estavam na Cochrane Library, 12 na Public Medline e 1 na PEDro. Os estudos evidenciaram que as pacientes apresentaram uma redução do índice glicêmico. Conclusão: Pôde-se concluir que o fisioterapeuta tem papel fundamental na prevenção e controle da DMG. Assim, contribuindo para uma melhor qualidade de vida para a gestante.

Palavras-chave: Eclampsia; Fisioterapia; Diabetes gestacional; Saúde da mulher.

\begin{abstract}
Introduction: Gestational Diabetes Mellitus (GDM) occurs when the body is not efficient at capturing enough glucose to meet extra needs during pregnancy, resulting in hyperglycemia of varying severity, resulting from the total or partial deficit of the hormone insulin. Objective: To perform a literature review on the importance of physiotherapists at the control end no prevention of Gestational Diabetes Mellitus. Methods: This is a literature review. Data were collected in the PEDro, PubMed and Cochrane Library databases. The selected descriptors were: Eclampsia, Physiotherapy, and Gestational Diabetes. The inclusion criteria were complete articles, published in national and international journals, and containing information that responded to the theme of the study. The exclusion criteria involved duplicate publications. Results and Discussions: In the crossing of the descriptors, 17 articles were found, of these, 4 were in the Cochrane Library, 12 in the Public Medline and 1 in PEDro. The studies showed that the patients showed a reduction in the glycemic index. Conclusion: It was concluded that the physiotherapist has a fundamental role in the prevention and control of GDM. Thus, contributing to a better quality of life for pregnant women.
\end{abstract}

Keywords: Eclampsia; Physical therapy specialty; Diabetes gestational; Women's health.

\begin{abstract}
Resumen
Introducción: La diabetes mellitus gestacional (DMG) ocurre cuando el cuerpo no es eficiente para capturar suficiente glucosa para realizar necesidades adicionales durante el embarazo, lo que resulta en una hiperglucemia de diversa gravedad, resultante del déficit total o parcial de la hormona insulina. Objetivo: Realizar una revisión de la literatura sobre la importancia del fisioterapeuta em nel control y prevención de Diabetes Mellitus Gestacional. Métodos: Esta es una revisión de la literatura. Los datos se recolectaron en las bases de datos PEDro, PubMed y Cochrane Library E. Los descriptores seleccionados fueron: Eclampsia, Fisioterapia y Diabetes gestacional. Los criterios de inclusión fueron artículos completos, publicados en revistas nacionales e internacionales, y que contuvieran información que respondiera a la temática del estudio. Los criterios de exclusión incluyeron publicaciones duplicadas. Resultados y Discusiones: En el cruce de los descriptores se encontraron 17 artículos, de los cuales 4 se encontraban en la Cochrane Library, 12 en la Public Medline y 1 en PEDro. Los estudios mostraron que los pacientes mostraron una reducción en el índice glucémico. Conclusión: Se concluyó que el fisioterapeuta tiene un papel fundamental en la prevención y control de la DMG. Contribuyendo así a una mejor calidad de vida de las mujeres embarazadas.
\end{abstract}

Palabras clave: Eclampsia; Fisioterapia; Diabetes gestacional; Salud de la mujer.

\section{Introdução}

O diabetes mellitus gestacional (DMG) ocorre quando o corpo não é eficiente para captar glicose suficiente para realizar as necessidades extras durante a gestação, fato que resulta em hiperglicemia, proveniente do déficit total ou parcial do hormônio insulina (Pontes, 2015 e Signer et al, 2012). 
Em uma gravidez habitual, a resistência ao hormônio insulina e o nível de glicemia pós-prandial (após uma refeição) aumentam a partir do segundo trimestre, devido ao efeito diabetogênico de um ou mais hormônios gestacionais secretados pela placenta, e o nível de glicose em jejum reduz durante o primeiro trimestre (Spaight, Gross, Horsch \& Puder, 2016 e Signer et al, 2012).

O DMG é uma complicação comum entre as gestantes, ocorrendo de 3 a $7 \%$ das gestações. A mortalidade materna representa um problema grave de saúde pública. Estima-se que 600 mil mulheres morram anualmente no mundo durante a gestação e o parto. E a Diabetes Melitus Gestacional está entre os fatores de risco que contribuem para tal estimativa (Cruz, Metring \& Carbone, 2015 e Martins et al, 2020).

Mulheres que apresentam o DMG são predispostas a desenvolverem a doença hipertensiva específica da gravidez, sendo mais propensas a terem um parto induzido (Junior, Souza, Agra, Filho \& Alves, 2016). A Doença Hipertensiva Específica da Gravidez pode ser identificada pelo aparecimento de edema pouco relevante nos membros inferiores, mas que pode evoluir para a eclâmpsia, fato que eleva o risco de mortalidade materno-fetal e parto prematuro (Signe et al., 2012; Merey, Marques \& Gimaraes, 2013; Brown et al., 2017).

A Diabetes Mellitus Gestacional é um fator de risco para o aparecimento da macrossomia, que é diagnosticada quando o recém-nascido nasce com peso igual ou superior a 4.000g (Brown et al., 2017). Tanto na prevenção quanto no tratamento do Diabetes Mellitus Gestacional, a fisioterapia tem um papel importante para a melhora da qualidade de vida dessa população (Bisneta et al. 2020; Silva, 2020).

Um programa de intervenção individualizado e bem planejado é importante para uma gestação saudável. É necessária uma boa avaliação a cada trimestre da gestação, para facilitar a adequação às alterações que ocorrem nesse período (Merey et al., 2013). Com essa informação, esse estudo tem como objetivo realizar uma revisão da literatura sobre a importância da Fisioterapia para o controle e prevenção do Diabetes Mellitus Gestacional.

\section{Metodologia}

Trata-se de uma revisão integrativa da literatura de caráter descritivo com abordagem qualitativa. O presente estudo foi construído respeitando as seguintes etapas: a) elaboração da pergunta norteadora; b) busca na literatura; c) coleta de dados; d) análise crítica dos estudos incluídos; e) discussão dos resultados; f) apresentação da revisão.

A pergunta que norteou o presente estudo foi a seguinte: "Qual a importância da fisioterapia para o controle do diabetes mellitus gestacional?" Qual o impacto da Fisioterapia na qualidade de vida dessa população? Foi realizado levantamento bibliográfico nas bases de dados Physiotherapy Evidence Database (PEDRo), Public Medline (PubMed) e Cochrane Library, sobre o assunto de interesse publicado no período de 2011 a 2020.

A seleção dos artigos foi realizada em novembro e dezembro de 2020, por todos os pesquisadores envolvidos de forma independentes, utilizando os descritores nos idiomas inglês e português.

Os descritores selecionados para a busca dos artigos foram: Fisioterapia, Eclampsia e Diabetes Gestacional, utilizando estratégia de busca o operador booleano AND. Os estudos foram selecionados inicialmente pela leitura de título e resumo. Em sequência foi realizada a leitura de texto completo, para garantia ou não da adequação aos critérios de inclusão. Foi realizada uma síntese de cada estudo, trazendo suas principais informações.

Os critérios de inclusão dos artigos da presente revisão foram: publicações completas em periódicos nacionais e internacionais, publicados entre os anos de 2011 a 2020; artigos que abordassem a temática do estudo, ou seja, estudos que evidenciaram o papel do Fisioterapeuta para o controle da Diabetes Gestacional. Os critérios de não inclusão envolveram as publicações duplicadas e artigos referentes à outra área de estudo. 
A análise dos dados extraídos foi realizada de forma descritiva, sendo identificadas informações quanto à localização do artigo, ano e periódico de publicação, autoria, objetivo, metodologia, resultados principais. A coleta dos dados foi realizada de acordo com a Figura 1.

Figura 1 - Fluxograma de busca e seleção de artigos nas bases de dados PEDRo, Pubmed e Cochrane

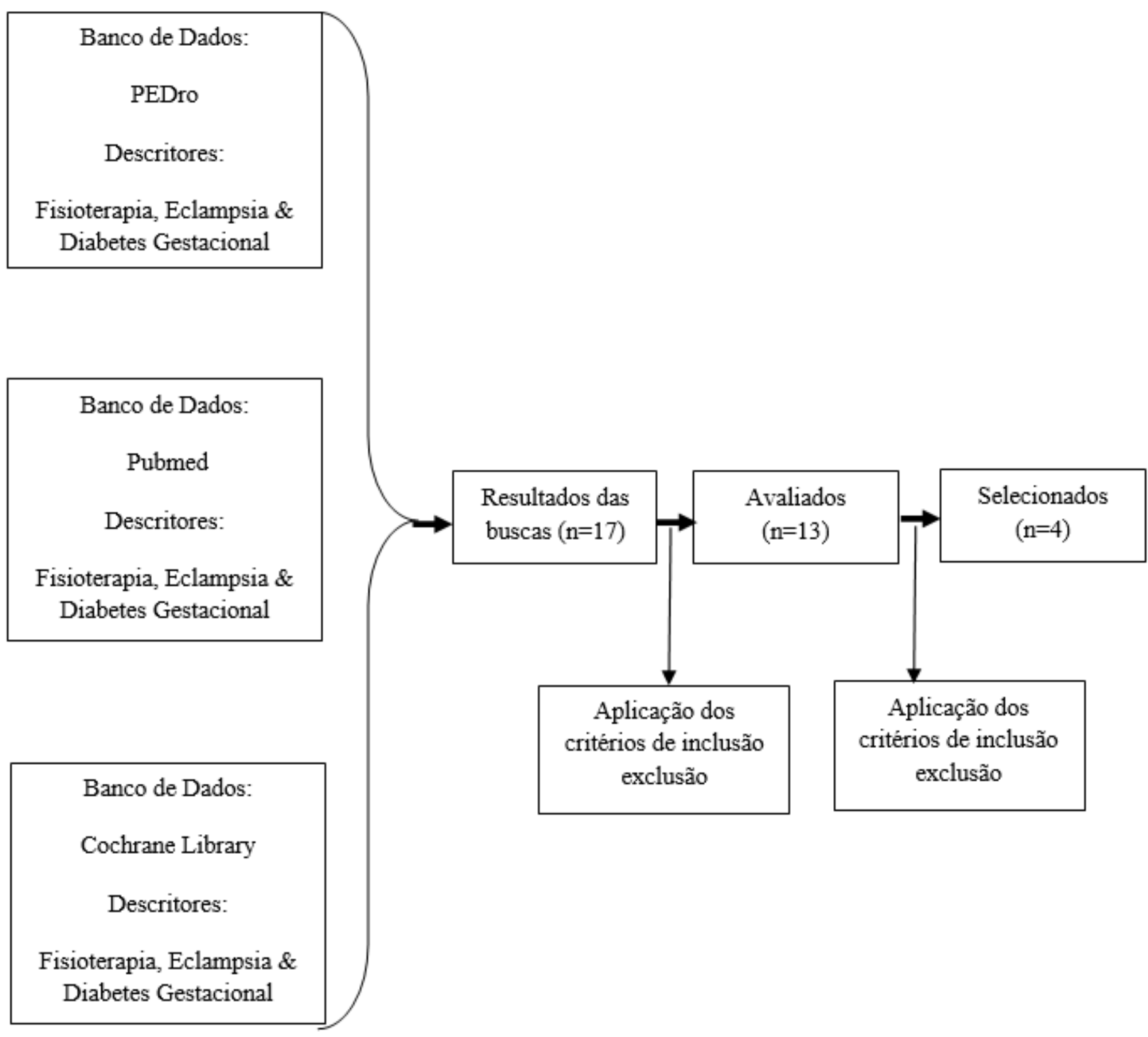

Fonte: Autores (2021).

\section{Resultados e Discussão}

Foram encontrados 17 artigos com os descritores selecionados, sendo 1 Physiotherapy Evidence Database, 12 no Public Medline e 4 no Cochrane Library. Destes estudos, 13 não atenderam aos critérios de elegibilidade para a realização deste estudo. Assim, foram incluídos apenas quatro artigos científicos que evidenciaram a atuação do Fisioterapeuta para o controle do Diabetes Mellitus Gestacional. 
Os principais recursos utilizados foram: Exercícios aeróbicos, fortalecimento muscular, treino de equilíbrio, exercícios domiciliares, alongamento muscular, exercícios respiratórios e relaxamento muscular.

Ao analisar e verificar o período de publicação dos artigos selecionados, o maior número de artigos publicados encontrados foram em 2013 com duas publicações; seguido de 2011 e 2012, com uma publicação para ambos os períodos. Os artigos selecionados para essa revisão são apresentados no Quadro 1.

Quadro 1 - Distribuição dos artigos selecionados.

\begin{tabular}{|c|c|c|c|}
\hline Autor e ano & Tipo de estudo & Principais Intervenções & Resultado \\
\hline $\begin{array}{c}\text { Stafne, Salvesen, Romundstad, } \\
\text { Eggeb } \varnothing, \text { Carlsen \& Mørkved } \\
\text { (2012) }\end{array}$ & $\begin{array}{l}\text { Estudo controlado e } \\
\text { randomizado }\end{array}$ & $\begin{array}{l}\text { Grupo intervenção }(\mathrm{n}=375) \text { : } \\
\text { exercícios aeróbicos, } \\
\text { fortalecimento muscular, treino } \\
\text { de equilíbrio, exercícios } \\
\text { domiciliares, } \\
\text { Grupo controle (n=327): cuidado } \\
\text { pré- natal sem a intervenção } \\
\text { Fisioterapêutica. }\end{array}$ & $\begin{array}{l}\text { O grupo de intervenção } \\
\text { apresentou menor índice } \\
\text { glicêmico. }\end{array}$ \\
\hline $\begin{array}{c}\text { Barakat, Pelaez, Lopez, Lucia } \\
\text { \& Ruiz (2013) }\end{array}$ & $\begin{array}{l}\text { Estudo controlado e } \\
\text { randomizado }\end{array}$ & $\begin{array}{c}\text { Grupo intervenção }(\mathrm{n}=210) \text { : } \\
\text { exercício 3x por semana,50-55 } \\
\text { min exercício aeróbico, } \\
\text { fortalecimento, flexibilidade e } \\
\text { dança. Grupo controle }(\mathrm{n}=210) \text { : } \\
\text { pré-natal usual que receberam as } \\
\text { aorientações a respeito dos efeitos } \\
\text { dos exercícios. }\end{array}$ & $\begin{array}{l}\text { O grupo de intervenção } \\
\text { apresentou menor nível de } \\
\text { glicose e redução no } \\
\text { peso. }\end{array}$ \\
\hline Pedrini \& Levone (2011) & Estudo de caso & $\begin{array}{l}\text { Estudo de caso }(\mathrm{n}=1) \text { : exercício } \\
\text { de mobilidade articular, exercício } \\
\text { respiratórios, alongamentos } \\
\text { passivos e ativos, orientações } \\
\text { sobre o posicionamento. }\end{array}$ & $\begin{array}{l}\text { Diminuição da frequência } \\
\text { respiratória, redução da } \\
\text { dor e dos níveis } \\
\text { glicêmicos após as } \\
\text { intervenções. }\end{array}$ \\
\hline $\begin{array}{l}\text { Merey, Marques, Matos \& } \\
\text { Guimaraões (2013) }\end{array}$ & $\begin{array}{c}\text { Estudo longitudinal e } \\
\text { quantitativo }\end{array}$ & $\begin{array}{c}9 \text { pacientes receberam o } \\
\text { alongamento muscular, exercícios } \\
\text { aeróbicos, exercícios } \\
\text { respiratórios, e relaxamento } \\
\text { muscular.. }\end{array}$ & $\begin{array}{l}\text { Menor índice glicêmico } \\
\text { após a finalização do } \\
\text { estudo. }\end{array}$ \\
\hline
\end{tabular}

Fonte: Autores (2021).

A partir dos resultados obtidos nos estudos revisados, verificou-se a redução dos níveis glicêmicos nas mulheres com DMG que participaram dos programas de intervenção. O estudo do tipo controlado e randomizado foi o mais utilizado para abordar a temática desse estudo. Apesar dessa informação, foi observado uma escassez sobre a temática abordada.

As mulheres com maior risco de desenvolver DMG são aquelas que têm o histórico de intolerância à glicose ou Diabetes Gestacional no passado. A partir dos 25 anos, a prevalência do desenvolvimento da DMG cresce. Raça e etnia também são fatores de risco importantes, geralmente a prevalência da doença é maior entre mulheres asiáticas (mesmo com IMC adequado), mulheres norte-africanas e hispânicas do que em mulheres brancas não hispânicas (Brown et al. 2017).

O sobrepeso pré-gravidez também é um forte fator de risco em todos os grupos étnicos; estudos recentes demonstraram um risco duas a nove vezes maior de DMG quando a obesidade aumenta da Classe I (IMC 30-34,9 $\mathrm{kg} / \mathrm{m}$ 2) para a Classe III (IMC> $40 \mathrm{~kg} / \mathrm{m}$ 2) (Spaight et al. 2016). 
A deficiência de vitamina $\mathrm{D}$ também entra no rol dos fatores de risco, mulheres com tal deficiência, têm um risco 3 a 7 vezes maior de desenvolver DMG em comparação com mulheres com nível de vitamina D suficiente (Spaight et al. 2016).

Outros fatores de risco podem ser citados, como: idade materna avançada, alta paridade, história familiar de diabetes mellitus e síndrome do ovário policístico (Brown et al. 2017). As modalidades terapêuticas na rotina de tratamento para DMG, obrigatoriamente inclui, uma equipe multidisciplinar a fim de aplicar programas de tratamento e prevenção que atenda cada paciente de forma individualizada (Spaight et al. 2016). Entre os profissionais, o Fisioterapeuta tem um papel importante, garantindo o bem estar e a qualidade de vida das gestantes.

No estudo de Stefne et el. (2012) usou-se os exercícios aeróbicos, fortalecimento muscular, treino de equilíbrio e exercícios domiciliares. Foi possível verificar a redução da resistência à insulina, através do rebaixamento do nível de glicemia de jejum. Igualmente ao estudo de Stefne et el. (2012), Barakat et al. (2013), utilizaram exercícios aeróbicos e fortalecimento muscular. Além desses recursos, o de Barakat et al, utilizou exercícios de mobilidade e dança. A redução dos níveis glicêmicos também foi encontrado no estudo de Barakat et al. (2013), e além disso, as pacientes apresentaram uma redução no peso materno.

Através da prática de exercícios de mobilidade articular, exercícios respiratórios, alongamentos e mudança de decúbito, Pedrini e Levone (2011), realizaram um estudo de caso de três semanas de intervenção. As pacientes apresentaram uma diminuição da dor e uma redução no nível glicêmico.

Através de um programa de exercícios físicos, de intensidade moderada, Merey et al. (2013), evidenciaram a importância do fisioterapeuta para o controle do Diabetes Mellitus Gestacional. Após a intervenção, foi encontrado uma diminuição dos índices glicêmicos, após 60 minutos de terapia. As pacientes também apresentaram uma mudança significativa no índice de saturação de oxigênio. Os exercícios respiratórios, alongamentos, exercícios aeróbicos e relaxamento por 4 semanas mostraram-se eficazes para essas pacientes.

Segundos os estudos revisados, os exercícios leves ou moderados, têm um papel importante na reabilitação de gestantes com o Diabetes Mellitus Gestacional. Visto que, o exercício diminui a quantidade de glicose no sangue, por aumentar a disponibilidade dos receptores de insulina e consequentemente reduz a intolerância à glicose através do condicionamento cardiovascular (Pedrini \& Levone, 2011).

O aumento do fluxo sanguíneo para os tecidos sensíveis à insulina, proporciona a redução dos níveis de ácidos graxos livres (Almeida et al. 2020). Até o momento, pouco se sabe sobre o fortalecimento muscular para o controle do diabetes mellitus gestacional, mas os estudos revisados mostraram-se eficazes e seguros para essas gestantes.

São contraindicados exercícios com cargas elevadas ou que favoreçam o aumento da pressão abdominal. Entende-se também que em alguns casos, o exercício físico de forma isolada não é suficiente para atingir o objetivo do controle glicêmico, fazendo-se necessária a insulinoterapia (Pedrini \& Levone, 2011). Sendo assim, a contribuição do fisioterapeuta é necessária para agregar a melhora da qualidade de vida da gestante. Tanto na prevenção quanto no tratamento do diabetes tipo 2 .

\section{Conclusão}

Diante de todas as informações expostas neste estudo, pode-se entender que o Diabetes Gestacional é um problema de saúde pública que afeta mulheres em todo o mundo. O fisioterapeuta atua na prevenção e no controle do Diabetes Gestacional, visto que, para um bom resultado se faz necessário um acompanhamento de forma individual, para atender as necessidades de cada gestante, assim, prevenindo futuros problemas de saúde e, consequentemente, melhorando a qualidade de vida. É recomendando mais pesquisas relacionadas ao tema abordado nesse estudo, para que assim os resultados sejam melhor disseminados e evidenciados para trazer uma maior atenção a gestante com o Diabetes Gestacional 


\section{Referências}

Almeida, P. T., Teixeira, Y., Barbosa, A. K. da silva, Lima, W. L., Fernandes, V. dos S., Sousa Junior, F. W. de, Silva, R. F. da, Lima, L. R., Pereira, T. da C., Barreto, J. A. P. S., Menezes, R. S. M. de, Guedes, I. C. P., Silva, C. L., Bezerra, P. de S., Silva, I. M. F. da, Beserra, T. L., Santos, S. M. S., Marques, R. M., Silva, P. N. da, Araújo, J. A. de, Santos Neto, H. P. dos, Russel, E. S., \& Trajano, J. A. (2020). Assistência nutricional e diabetes mellitus gestacional: uma revisão integrativa de literatura. Research, Society and Development, 9(7), e640974592. https://doi.org/10.33448/rsd-v9i7.459

Barakat, R., Pelaez, M., Lopez, C., Lucia, A., \& Ruiz, J. R. (2013). Exercise during pregnancy and gestational diabetes-related adverse effects: a randomised controlled trial. British journal of sports medicine, 47(10), 630-636. https://doi.org/10.1136/bjsports-2012-091788

Bisneta, I. P. da S., Beltrão, S. da S. A., Lima, F. L. O., Silva, C. D. C. M., \& Silva, M. V. C. M. (2020). Alterações fisiológicas na captação de glicose pelo GLUT-4 no Diabetes Mellitus Gestacional. Research, Society and Development, 9(7), e857974783. https://doi.org/10.33448/rsd-v9i7.4783

Brown, J., Alwan, N. A., West, J., Brown, S., McKinlay, C. J., Farrar, D., \& Crowther, C. A. (2017). Lifestyle interventions for the treatment of women with gestational diabetes. The Cochrane database of systematic reviews, 5(5), CD011970. https://doi.org/10.1002/14651858.CD011970.pub2

Cruz, F. C. A., Metring, N. L., \& Carbone, É. S. M (2015). Exercício resistido como abordagem terapêutica da fisioterapia no diabetes mellitus gestacional revisão sistemática. REVISTAINSPIRAR movimento \& saúde, v. 7, n1.

Martins, G. K. F., Carreli, G. Z., Ferreto, L. E. D., Dalmolin, B. L., Vandresen, D. F., Vicentini, G. E., de Araújo, A. K., Righi, M. G., \& Wendt, G. W. (2020). Prevalence and associated factors of gestational diabetes mellitus in a high complexity hospital. Research, Society and Development, 9(8), e173985541. https://doi.org/10.33448/rsd-v9i8.5541

Merey, L., Marques, C., de Matos, H., \& Guimarães, T. (2013). Aplicação de um programa de exercício físico em gestantes diabéticas. Fisioterapia Brasil, 14(5), 338 - 343. http://dx.doi.org/10.33233/fb.v14i5.415

Pedrini, A., \& Levone, B., R. (2011). Fisioterapia no Diabetes Mellitus Gestacional Revista Brasileira de Ciências da Saúde, 9(28). https://doi.org/10.13037/rbcs.vol9n28.1371

Portes, L. (2015). Abordagem do fisioterapeuta no diabetes mellitus: revisão de literatura. Arquivos De Ciências Da Saúde, 22(3), 9-14. 10.17696/23183691.22.3.2015.37

Silva Junior, J. R. da, et al (2016). Diabetes mellitus gestacional: importância da produção de conhecimento. Revista Brasileira de Saúde Materno Infantil, 16(2), 85-87. https://doi.org/10.1590/1806-93042016000200001

Silva, M. E. da, et al (2020). Benefits of physical exercise in pregnancy: an integrative review. Research, Society and Development, 9(11), e83291110401. https://doi.org/10.33448/rsd-v9i11.10401

Spaight, C., Gross, J., Horsch, A., \& Puder, J. J. (2016). Gestational Diabetes Mellitus. Endocrine development, 31, 163-178. https://doi.org/10.1159/000439413

Stafne, S. N., Salvesen, K. Å., Romundstad, P. R., Eggebø, T. M., Carlsen, S. M., \& Mørkved, S. (2012). Regular exercise during pregnancy to prevent gestational diabetes: a randomized controlled trial. Obstetrics and gynecology, 119(1), 29-36. https://doi.org/10.1097/AOG.0b013e3182393f86 